\title{
An Approximate Analytical Approach for Systems of Fredholm Integro-Differential Equations of Fractional Order
}

\author{
Mudaffer Alnobani ${ }^{1, *}$ Omar Abu Al Yaqin ${ }^{2}$ \\ ${ }^{1,2}$ Department of Mathematics and Statistics, Jordan University of Science \& Technology, \\ Irbid, 22110, Jordan \\ ${ }^{1}$ maalnobani18@sci.just.edu.jo \\ 2 omabualyaqin18@sci.just.edu.jo \\ *Corresponding author
}

Received: April 22, 2021. Revised: May 25, 2021. Accepted: May 27, 2021. Published: June 3, 2021.

\begin{abstract}
A new technique for solving a system of fractional Fredholm integro-differential equations (IDEs) is introduced in this manuscript. Furthermore, we present a review for the derivation of the residual power series method (RPSM) to solve fractional Fredholm IDEs in the paper done by Syam, as well as, corrections to the examples mentioned in that paper. The numerical results demonstrated the new technique's applicability, efficacy, and high accuracy in dealing with these systems. On the other hand, a comparison has been done between the two schemes using the two corrected examples in addition to a problem that had been solved in many previous studies, and the results of these studies were compared with the new technique and RPSM. The comparison demonstrated clear superiority of our method over the RPSM for solving this class of equations. Moreover, they dispel the misconception that the RPSM works effectively on fractional Fredholm IDEs as mentioned in the paper done by Syam, whereas two problems solved by the RPSM produced an unaccepted error. Also, the comparison with the previous studies indicates the importance of the new method in dealing with the fractional Fredholm IDEs despite its simplicity, ease of use, and negligible computational time.
\end{abstract}

Keywords- Caputo derivative, residual power series method, fractional power series, fractional Fredholm integro-differentional equations.

\section{INTRODUCTION}

During the last century, the fractional calculus was used to model many natural phenomena in different fields including natural sciences, engineering, and numerical analysis such as electric-circuit analysis [1], earthquake [2], fluid-dynamic traffic [3], measurement of viscoelastic material properties [4], control theory [5], solid mechanics models [6] and others.

The fractional integro-differential equations (FIDEs) have a fundamental role in modeling many phenomena in some of the above-mentioned disciplines such as presenting the control process and the dynamics of different systems, Electric circuit analysis, the activity of synaptically coupled networks of excitatory and inhibitory neurons, and viscoelastic material dynamics [7, 8, 9]. Many techniques were applied to find approximate solutions for FIDEs since the majority of them do not have exact solution, or it is difficult to calculate.

A lot of methods have been developed to find approximate solutions for single fractional Fredholm integro-differential equations (IDEs). In the lines that follow, some of these approaches will be discussed. For instance, Saeedi, Moghadam, Mollahasani and Chuev [10] utilized a new approach to solve nonlinear fractional Fredholm IDEs that relied on the Sine and Cosine wavelets. In [11] Samimi and Saeedi introduced a new technique, based on the Homotopy Perturbation method, to approximate the solution for a class of nonlinear Fredholm IDEs of fractional order. Saeedi used Block Pulse functions and Haar wavelets to solve a nonlinear fractional Fredholm IDEs in [12]. Amit Setia, Yucheng Liu and Vatsala [13] find approximate solutions for linear Fredholm FIDEs by using the Chebyshev wavelets method. Darweesh, Alquran and Aghzawi [14] developed a new algorithm, based on Haar wavelets, to find approximate solutions for a class of twodimensional fractional Fredholm IDEs, and they introduced a modification to that method by employing the Laplace transform.

In the last fifteen years, a lot of attention has been paid to develop new techniques that solve systems of fractional IDEs due to their repeated appearance in many disciplines such as engineering and chemistry [15, 16]. Some of these techniques will be listed in the lines that follow. For instance, Qaralleh and Momani [17] used the Adomian decomposition approach to approximate the solutions for linear and nonlinear coupled systems of fractional IDEs. Sweilam and Khader [18] utilized a new approach, based on the Chebyshev pseudospectral method, to find approximate solutions for a coupled system of linear and nonlinear fractional Volterra IDEs in one-dimensional space. Khan and Khalil [19] proposed operational matrices based on the shifted Legendre polynomials to generate approximate solutions for a coupled system of onedimensional linear fractional Fredholm IDEs. Wang, Xu, Wei and Xie [20] used a new technique that relies on Bernoulli wavelets to approximate the solution of coupled systems of nonlinear fractional IDEs of Volterra type. Mahdy [21] in- 
troduced a new technique with the aid of the least squares method and Hermite method to produce approximate solutions for a coupled system of one-dimensional linear Fredholm FIDEs. Mohammed and Malik [22] utilized a modified computational algorithm to solve a coupled system of onedimensional linear fractional Volterra IDEs. Xie, Wang, Ren, Zhang and Quan[23] employed the Haar wavelets method to find approximate solutions for a coupled system of onedimensional fractional IDEs of Volterra type.

In our framework, we are concerned with studying a system of fractional Fredholm IDEs of the following form:

$$
\begin{array}{cc}
D^{\alpha_{1}} u_{1}(x)= & f_{1}(x)+\int_{0}^{1}\left(u_{1}(t) K_{1,1}(x, t)+\right. \\
& \left.u_{2}(t) K_{1,2}(x, t)+\ldots+u_{n}(t) K_{1, n}(x, t)\right) d t \\
D^{\alpha_{2}} u_{2}(x)= & f_{2}(x)+\int_{0}^{1}\left(u_{1}(t) K_{2,1}(x, t)+\right. \\
& \left.u_{2}(t) K_{2,2}(x, t)+\ldots+u_{n}(t) K_{2, n}(x, t)\right) d t \\
\vdots & \\
D^{\alpha_{n}} u_{n}(x)= & f_{n}(x)+\int_{0}^{1}\left(u_{1}(t) K_{n, 1}(x, t)+\right. \\
& \left.u_{2}(t) K_{n, 2}(x, t)+\ldots+u_{n}(t) K_{n, n}(x, t)\right) d t,
\end{array}
$$

with

$$
\alpha_{i} \in(0,1] \forall i=1,2,3, . ., n \text {, and } x, t \in[0, a] \text {, }
$$

subject to the initial conditions

$$
u_{i}(0)=c_{i, 0} \forall i=1,2,3, \cdots, n
$$

where the fractional derivatives are in the Caputo sense, $K_{i, j}(x, t)$ are arbitrary continuous kernels over $[0, a] \forall i, j=$ $1,2,3, . ., n, u_{i}(x)$ are analytic unknown functions to be calculated with $a>0 \forall i=1,2,3, . ., n$, a is the convergent radius, and $f_{i}(x)$ are smooth functions $\forall i=1,2,3, \ldots, n$.

Our primary objectives of this work are to first find an analytic-numeric solution for system (1) using a modified scheme that is similar to the residual power series method (RPSM). Second, we review paper [24], which employed the RPSM to solve the single form of system 1 , and correct the findings presented in that paper. Finally, we compare the proposed technique with the RPSM.

The residual power series method was introduced and utilized by Abu Arqub in [25] for solving fuzzy differential equations. The RPSM was applied to solve first-order initial value problems in [26] by Al-Smadi. Also, some papers solved several kinds of partial differential equations with fractional order depending on the use of RPSM [27, 28, 29]. Komashynska, Al-Smadi, Ateiwi, and Al-Obaidy [30] used the RPSM to find approximate solutions for a system of Fredholm integral equations. Moreover, the RPSM was utilized to solve several types of fractional IDEs, we mention some of them in the lines that follow. Alshammari, Al-Smadi, Hashim, and Alias [31] solved Volterra IDEs with fractional order using the RPSM, and they solved fractional mixed IDEs using the same method in [32].

The rest of this article is composed as follows; In section III two background topics are revised concerning fractional derivative and power series generalization to fractional power series (FPS). In section III, we present the modified scheme that deals with the system (1). The performance of the scheme is illustrated and investigated in section IV] with two numerical examples to prove its efficiency. In section $\mathrm{V}$, we correct the work done, in employing the RPSM to find the recursive formula for the coefficients of the unknown function, in paper [24]. Moreover, we correct the first two examples written in that paper and solve them by the two methods with a brief comparison between the two methods in solving the single equation form of the system (1). In section VI, we comment and discuss the most salient points that resulted from subsection V.B. Moreover, we mention the features of the proposed scheme in section III and its limitations. Finally, we conclude this article with brief observations and conclusions in section VII

\section{PRELIMINARIES AND BASIC DEFINITIONS}

\section{II.A Fractional Derivative}

Throughout this section, we review the Caputo fractional derivative definition and some basic results about it. In this framework, we use the fractional derivatives described in $\mathrm{Ca}-$ puto's definition from a variety of fractional derivative definitions.

Definition 2.1.1 [33] (Caputo fractional derivative). Suppose that $\alpha>0, x>0, \alpha, x \in \mathbb{R}$. The operator of fractional calculus:

$$
D_{x}^{\alpha} f(x)=\left\{\begin{array}{rr}
\frac{1}{\Gamma(n-\alpha)} \int_{0}^{x} f^{(n)}(\xi)(x-\xi)^{n-\alpha-1} d \xi, & \text { if } \\
\frac{d^{n}}{d x^{n}} f(x), & n-1<\alpha<n \in \mathbb{N}, \\
& \alpha=n \in \mathbb{N} .
\end{array}\right.
$$

is called the Caputo fractional derivative or Caputo differential operator of fractional calculus of order $\alpha$.

Theorem 2.1.2 [33]. Let $n-1<\alpha<n, n \in \mathbb{N}, \alpha, \beta \in \mathbb{R}$, and $\lambda, z \in \mathbb{C}$. Let $f(x)$ and $g(x)$ be such that both $D_{x}^{\alpha} f(x)$ and $D_{x}^{\alpha} g(x)$ exist. Then

1) Linearity: $D_{x}^{\alpha}(\lambda f(x)+g(x))=\lambda D_{x}^{\alpha} f(x)+D_{x}^{\alpha} g(x)$.

2) $D_{x}^{\alpha} c=0$ for any constant $c \in \mathbb{R}$.

3)

$$
D_{x}^{\alpha} x^{p}=\left\{\begin{array}{l}
\frac{\Gamma(p+1)}{\Gamma(p-\alpha+1)} x^{p-\alpha}, \text { if } \\
n-1<\alpha<n, p>n-1, p \in \mathbb{R} \\
0, \text { if } \quad n-1<\alpha<n, p \leq n-1, p \in \mathbb{N}_{0} .
\end{array}\right.
$$

4) The Caputo fractional derivative of the exponential function has the following form:

$$
D_{x}^{\alpha} e^{\lambda x}=\sum_{k=0}^{\infty} \frac{\lambda^{k+n} x^{k+n-\alpha}}{\Gamma(k+1+n-\alpha)}=\lambda^{n} x^{n-\alpha} E_{1, n-\alpha+1}(\lambda x) .
$$

where

$$
E_{\alpha, \beta}(z)=\sum_{k=0}^{\infty} \frac{z^{k}}{\Gamma(k \alpha+\beta)}
$$

where $\alpha, \beta>0, \alpha, \beta \in \mathbb{R}, z \in \mathbb{C}$. is generalized MittagLeffler function. 


\section{II.B Fractional Power Series}

In this section, we recall some fundamental definitions and theorems concerning power series generalization to fractional power series (FPS) [34].

Definition 2.2.1 [34]. A power series representation of the form

$$
\sum_{n=0}^{\infty} c_{n}(x-a)^{n \alpha}=c_{0}+c_{1}(x-a)^{\alpha}+c_{2}(x-a)^{2 \alpha}+\cdots
$$

where $0 \leq m-1<\alpha \leq m$ and $x \geq a$ is called a fractional power series about $a$, where $x$ is a variable and $c_{n}$ 's are the constants called the coefficients of the series.

Theorem 2.2.2 [34]. Suppose that $f$ has a FPS representation at $a$ of the form:

$$
f(x)=\sum_{n=0}^{\infty} c_{n}(x-a)^{n \alpha}, 0 \leq m-1<\alpha \leq m, a \leq x<a+R
$$

If $f(x) \in C[a, a+R)$ and $D^{n \alpha} f(x) \in C(a, a+R)$ for $n=$ $0,1,2, \cdots$, then the coefficients $c_{n}$ in equation (3) will take the form $c_{n}=\frac{D^{n \alpha} f(a)}{\Gamma(n \alpha+1)}$, where $D^{n \alpha}=D^{\alpha} \cdot D^{\alpha} \cdots D^{\alpha} \quad(n-$ times $)$.

\section{THE METhodology OF THE PROPOSED METHOD}

The purpose of this section is to obtain an analyticnumeric solution based on the fractional power series expansion for a system of Fredholm fractional IDEs subject to certain initial conditions in the form of (1).

We expand the solution to the system (1) as fractional power series about $x=0$

$$
\begin{gathered}
u_{1}(x)=\sum_{j=0}^{\infty} c_{1, j} \frac{x^{j \alpha_{1}}}{\Gamma\left(j \alpha_{1}+1\right)}=u_{1}(x, k)+R_{1}(x, k+1) \\
u_{2}(x)=\sum_{j=0}^{\infty} c_{2, j} \frac{x^{j \alpha_{2}}}{\Gamma\left(j \alpha_{2}+1\right)}=u_{2}(x, k)+R_{2}(x, k+1) \\
\vdots \\
u_{n}(x)=\sum_{j=0}^{\infty} c_{n, j} \frac{x^{j \alpha_{n}}}{\Gamma\left(j \alpha_{n}+1\right)}=u_{n}(x, k)+R_{n}(x, k+1) .
\end{gathered}
$$

Where $u_{i}(x, k)=\sum_{j=0}^{k} c_{i, j} \frac{x^{j \alpha_{i}}}{\Gamma\left(j \alpha_{i}+1\right)}$ is the k-th truncated series of $u_{i}(x)$, and $R_{i}(x, m)=\sum_{j=m}^{\infty} c_{i, j} \frac{x^{j \alpha_{i}}}{\Gamma\left(j \alpha_{i}+1\right)}$. We require that $R_{i}(x, m)$ as small as negligible for $m \geq k+1$ on the interval $x \in(0,1)$ for all $i=1,2, \cdots, n$. Accordingly, we replace each $u_{i}(x)$ by the $k-t h$ truncated series $u_{i}(x, k)$ for all $i=1,2, \cdots, n$ in (1). Now, we aim to find the $k-t h$ approximation labeled as $u_{i}(x, k)$ for all $i=1,2, \cdots, n$ to the system (1), which can be summarized in the following steps:

Step 1: Consider system (1) after replacing each $u_{i}(x)$ by the $k-t h$ truncated series $u_{i}(x, k)$ for all $i=1,2, \cdots, n$, we obtain:

$$
\begin{aligned}
D^{\alpha_{1}} u_{1}(x, k) & =f_{1}(x)+\int_{0}^{1}\left(u_{1}(t, k) K_{1,1}(x, t)\right. \\
+ & \left.u_{2}(t, k) K_{1,2}(x, t)+\ldots+u_{n}(t, k) K_{1, n}(x, t)\right) d t \\
D^{\alpha_{2}} u_{2}(x, k) & =f_{2}(x)+\int_{0}^{1}\left(u_{1}(t, k) K_{2,1}(x, t)\right. \\
+ & \left.u_{2}(t, k) K_{2,2}(x, t)+\ldots+u_{n}(t, k) K_{2, n}(x, t)\right) d t \\
& \vdots \\
D^{\alpha_{n}} u_{n}(x, k) & =f_{n}(x)+\int_{0}^{1}\left(u_{1}(t, k) K_{n, 1}(x, t)\right. \\
+ & \left.u_{2}(t, k) K_{n, 2}(x, t)+\ldots+u_{n}(t, k) K_{n, n}(x, t)\right) d t
\end{aligned}
$$

Step 2: For all $m=1,2, \cdots, k$, we apply $\left.D^{(m-1) \alpha_{i}}\right|_{x=0}$ for all $i=1,2, \cdots, n$ on both sides of the equations of the system (5) as follows:

$$
\begin{gathered}
\left.D^{m \alpha_{1}} u_{1}(x, k)\right|_{x=0}=D^{(m-1) \alpha_{1}} f_{1}(0)+ \\
\int_{0}^{1} u_{1}(t, k) D^{(m-1) \alpha_{1}} K_{1,1}(0, t) d t \\
+\int_{0}^{1} u_{2}(t, k) D^{(m-1) \alpha_{1}} K_{1,2}(0, t) d t+ \\
\ldots+\int_{0}^{1} u_{n}(t, k) D^{(m-1) \alpha_{1}} K_{1, n}(0, t) d t \\
\left.D^{m \alpha_{2}} u_{2}(x, k)\right|_{x=0}=D^{(m-1) \alpha_{2}} f_{2}(0)+ \\
\int_{0}^{1} u_{1}(t, k) D^{(m-1) \alpha_{2}} K_{2,1}(0, t) d t \\
+\int_{0}^{1} u_{2}(t, k) D^{(m-1) \alpha_{2}} K_{2,2}(0, t) d t+ \\
\ldots+\int_{0}^{1} u_{n}(t, k) D^{(m-1) \alpha_{2}} K_{2, n}(0, t) d t \\
\vdots \\
\left.D^{m \alpha_{n}} u_{n}(x, k)\right|_{x=0}=D^{(m-1) \alpha_{n}} f_{n}(0)+ \\
\int_{0}^{1} u_{1}(t, k) D^{(m-1) \alpha_{n}} K_{n, 1}(0, t) d t+ \\
\int_{0}^{1} u_{2}(t, k) D^{(m-1) \alpha_{n}} K_{n, 2}(0, t) d t+ \\
\ldots \int_{0}^{1} u_{n}(t, k) D^{(m-1) \alpha_{n}} K_{n, n}(0, t) d t
\end{gathered}
$$

for all $m=1,2, \cdots, k$.

where $D^{m \alpha}=D^{\alpha} \cdot D^{\alpha} \cdots D^{\alpha} \quad(m-t$ times $)$.

Step 3: The previous step yields $k \times n$ equations. Moreover, we use the initial conditions given in (1) to find the values of $c_{i, 0}, \forall i=1,2, \cdots, n$ by substituting 0 in each equation of the system (4). Finally, we solve the previous $k \times n$ equations for $c_{i, j}$ for all $i=1,2, \cdots, n, j=1,2, \cdots, k$ to obtain our approximate solution to the system $(1)$ : $u(x) \approx\left(u_{1}(x, k), u_{2}(x, k), \cdots, u_{n}(x, k)\right)$.

\section{NUMERICAL EXAMPLES}

Two numerical examples are provided in this section, to demonstrate the efficiency of the proposed scheme. 
Example 1: Consider the following system of linear fractional Fredholm IDEs:

$$
\begin{aligned}
& D^{\frac{1}{2}} u_{1}(x)=f_{1}(x)+\int_{0}^{1} x u_{2}(t) d t \\
& D^{\frac{1}{2}} u_{2}(x)=f_{2}(x)+\int_{0}^{1}\left(t u_{1}(t)+\sqrt{x} u_{2}(t)\right) d t,
\end{aligned}
$$

subject to the initial condition $u_{1}(0)=0$ and $u_{2}(0)=1$, where

$$
\begin{aligned}
& f_{1}(x)= \sqrt{x} \sum_{j=0}^{\infty} \frac{(2 i x)^{j}\left(1+(-1)^{j}\right)}{\Gamma\left(j+\frac{3}{2}\right)}-\frac{1}{4} x \sin (4) \\
& f_{2}(x)=2 i \sqrt{x} \sum_{j=0}^{\infty} \frac{(4 i x)^{j}\left(1-(-1)^{j}\right)}{\Gamma\left(j+\frac{3}{2}\right)} \\
& \quad+\frac{1}{4}(2 \cos (2)-\sin (2)-\sqrt{x} \sin (4)),
\end{aligned}
$$

the exact solution is $u_{1}(x)=\sin (2 x), u_{2}(x)=\cos (4 x)$, and $i=\sqrt{-1}$.

We expand the solution to the system $(6)$ as fractional power series representation about $x=0$ of the form

$$
\begin{aligned}
& u_{1}(x)=\sum_{j=0}^{\infty} c_{j} \frac{x^{\frac{j}{2}}}{\Gamma\left(\left(\frac{j}{2}\right)+1\right)} \\
& u_{2}(x)=\sum_{j=0}^{\infty} b_{j} \frac{x^{\frac{j}{2}}}{\Gamma\left(\left(\frac{j}{2}\right)+1\right)} .
\end{aligned}
$$

Now, we apply the steps mentioned in section III by first replacing each $u_{l}(x)$ by the $k-t h$ truncated series $u_{l}(x, k)$ in system (6) for all $l=1,2$. Accordingly, we have

$$
\begin{aligned}
& D^{\frac{1}{2}} u_{1}(x, k)=f_{1}(x)+\int_{0}^{1} x u_{2}(t, k) d t \\
& D^{\frac{1}{2}} u_{2}(x, k)=f_{2}(x)+\int_{0}^{1}\left(t u_{1}(t, k)+\sqrt{x} u_{2}(t, k)\right) d t .
\end{aligned}
$$

Here, we write down some calculations needed for step 2:

1)

$$
\begin{aligned}
& D^{\frac{1}{2}(n)} f_{1}(x)= \sum_{j=\beta_{n}}^{\infty} \frac{(2 i)^{j}\left(1+(-1)^{j}\right) x^{j-\frac{n-1}{2}}}{\Gamma\left(j-\frac{n-3}{2}\right)}, \forall n \geq 3 . \\
& \text { Where } \\
& \beta_{n}= \begin{cases}\frac{n}{2} & \text { if } n=2 m, m=2,3,4 \cdots \\
\frac{n-1}{2} & \text { if } n=2 m-1, m=2,3,4 \cdots\end{cases}
\end{aligned}
$$

2) From note number one, we conclude that:

$$
D^{\frac{1}{2}(n)} f_{1}(0)= \begin{cases}0 & \text { if } n=2 m, m=2,3,4 \cdots \\ 0 & \text { if } n=2 m-1, m=2,4,6 \cdots \\ 2^{\frac{n+1}{2}} \times i^{\frac{n-1}{2}} & \text { if } n=2 m-1, m=3,5,7 \cdots\end{cases}
$$

3)

$$
\begin{gathered}
D^{\frac{1}{2}(n)} f_{2}(x)=\sum_{j=\beta_{n}}^{\infty} \frac{(2)^{2 j+1} i^{j+1}\left(1-(-1)^{j}\right) x^{j-\frac{n-1}{2}}}{\Gamma\left(j-\frac{n-3}{2}\right)}, \\
\forall n \geq 2 . \text { Where } \\
\beta_{n}= \begin{cases}\frac{n}{2} & \text { if } n=2 m, m=1,2,3,4 \cdots \\
\frac{n-1}{2} & \text { if } n=2 m-1, m=2,3,4 \cdots\end{cases}
\end{gathered}
$$

4) From note number three, we conclude that:

$$
D^{\frac{1}{2}(n)} f_{2}(0)= \begin{cases}0 & \text { if } n=2 m, m=1,2,3,4 \cdots \\ 0 & \text { if } n=2 m-1, m=3,5,7 \cdots \\ 2^{n+1} \times i^{\frac{n+1}{2}} & \text { if } n=2 m-1, m=2,4,6 \cdots\end{cases}
$$

5)

$$
\left.D^{\frac{1}{2}(n)}\left(\int_{0}^{1} x u_{2}(t, k) d t\right)\right|_{x=0}=0, \quad \forall n \geq 3 .
$$

6)

$$
\left.D^{\frac{1}{2}(n)}\left(\int_{0}^{1}\left(t u_{1}(t, k)+\sqrt{x} u_{2}(t, k)\right) d t\right)\right|_{x=0}=0, \forall n \geq 2 .
$$

Using the initial conditions given in this example by substituting 0 in (7), we find that $c_{0}=0$ and $b_{0}=1$. Now we write the $k \times 2$ equations as mentioned in step 2 of section 3 as follows:

For $m=1$, we have:

$$
\begin{aligned}
c_{1} & =0 \\
b_{1}-\frac{1}{4}(2 \cos (2)-\sin (2))-\sum_{j=0}^{k} c_{j} \frac{2+j}{2 \Gamma\left(\frac{j}{2}+3\right)} & =0 .
\end{aligned}
$$

For $m=2$, we have:

$$
\begin{aligned}
c_{2} & =2 \\
b_{2}+\frac{\sqrt{\pi} \sin (4)}{8}-\sum_{j=0}^{k} \frac{\sqrt{\pi} b_{j}}{2 \Gamma\left(\frac{j}{2}+2\right)} & =0 .
\end{aligned}
$$

For $m=3$, we have:

$$
\begin{aligned}
c_{3}+\frac{\sin (4)}{4}-\sum_{j=0}^{k} \frac{b_{j}}{\Gamma\left(\frac{j}{2}+2\right)} & =0 \\
b_{3} & =0 .
\end{aligned}
$$

Continuing in this process and using the calculations mentioned from 1 to 6 above we conclude the following:

$$
\begin{gathered}
c_{n+1}= \begin{cases}0 & \text { if } n=2 m, m=2,3,4 \cdots \\
0 & \text { if } n=2 m-1, m=2,4,6 \cdots \\
2^{\frac{n+1}{2}} \times i^{\frac{n-1}{2}} & \text { if } n=2 m-1, m=3,5,7 \cdots\end{cases} \\
b_{n+1}= \begin{cases}0 & \text { if } n=2 m, m=1,2,3,4 \cdots \\
0 & \text { if } n=2 m-1, m=3,5,7 \cdots \\
2^{n+1} \times i^{\frac{n+1}{2}} & \text { if } n=2 m-1, m=2,4,6 \cdots\end{cases}
\end{gathered}
$$

Now, we sum up the work done above. The unknown coefficients are $b_{1}, b_{2}$, and $c_{3}$. We can easily determine the value of these unknown coefficients, by solving the above equations for them, once we choose the level of approximation (the value of $k$ ) we want. As $k$ gets larger, the approximation gets better.

If we choose $k=100$. Then we find the values of $b_{1}, b_{2}$, and $c_{3}$ by solving $8 / 9[10: 11$ for them. We conclude that $c_{3} \approx-1.19409 \times 10^{-38}, b_{1} \approx-2.56646 \times 10^{-39}$, and $b_{2} \approx$ $1.05824 \times 10^{-38}$. 
TABLE I. Comparison between the exact solution $u_{1}(x)$ and the approximate solution $u_{1}(x, 100)$ together with the absolute errors at some points in $[0,1]$ for Example 1.

\begin{tabular}{||c|c|c|c||}
\hline$x$ & $u_{1}(x)[$ Exact $]$ & $u_{1}(x, 100)[$ Approximate $]$ & $u_{1}(x)-u_{1}(x, 100)$ \\
\hline 0.0 & 0.0 & 0.0 & 0.0 \\
\hline 0.1 & $1.986693308 \times 10^{-1}$ & $1.986693308 \times 10^{-1}$ & $2.77556 \times 10^{-17}$ \\
\hline 0.2 & $3.894183423 \times 10^{-1}$ & $3.894183423 \times 10^{-1}$ & $5.55112 \times 10^{-17}$ \\
\hline 0.3 & $5.646424734 \times 10^{-1}$ & $5.646424734 \times 10^{-1}$ & 0.0 \\
\hline 0.4 & $7.173560909 \times 10^{-1}$ & $7.173560909 \times 10^{-1}$ & $1.11022 \times 10^{-16}$ \\
\hline 0.5 & $8.414709848 \times 10^{-1}$ & $8.414709848 \times 10^{-1}$ & 0.0 \\
\hline 0.6 & $9.32039086 \times 10^{-1}$ & $9.32039086 \times 10^{-1}$ & $1.11022 \times 10^{-16}$ \\
\hline 0.7 & $9.8544973 \times 10^{-1}$ & $9.8544973 \times 10^{-1}$ & 0.0 \\
\hline 0.8 & $9.99573603 \times 10^{-1}$ & $9.99573603 \times 10^{-1}$ & 0.0 \\
\hline 0.9 & $9.738476309 \times 10^{-1}$ & $9.738476309 \times 10^{-1}$ & 0.0 \\
\hline 1.0 & $9.092974268 \times 10^{-1}$ & $9.092974268 \times 10^{-1}$ & 0.0 \\
\hline
\end{tabular}

TABLE II. Comparison between the exact solution $u_{2}(x)$ and the approximate solution $u_{2}(x, 100)$ together with the absolute errors at some points in $[0,1]$ for Example 1.

\begin{tabular}{||c|c|c|c||}
\hline$x$ & $u_{2}(x)[$ Exact $]$ & $u_{2}(x, 100)[$ Approximate $]$ & $\left|u_{2}(x)-u_{2}(x, 100)\right|$ \\
\hline 0.0 & 1.0 & 1 & 0.0 \\
\hline 0.1 & $9.21060994 \times 10^{-1}$ & $9.21060994 \times 10^{-1}$ & $1.11022 \times 10^{-16}$ \\
\hline 0.2 & $6.967067093 \times 10^{-1}$ & $6.967067093 \times 10^{-1}$ & 0.0 \\
\hline 0.3 & $3.623577545 \times 10^{-1}$ & $3.623577545 \times 10^{-1}$ & $5.55112 \times 10^{-17}$ \\
\hline 0.4 & $-2.91995223 \times 10^{-2}$ & $-2.91995223 \times 10^{-2}$ & $4.51028 \times 10^{-17}$ \\
\hline 0.5 & $-4.161468365 \times 10^{-1}$ & $-4.161468365 \times 10^{-1}$ & $5.55112 \times 10^{-17}$ \\
\hline 0.6 & $-7.373937155 \times 10^{-1}$ & $-7.373937155 \times 10^{-1}$ & $2.22045 \times 10^{-16}$ \\
\hline 0.7 & $-9.422223407 \times 10^{-1}$ & $-9.422223407 \times 10^{-1}$ & $1.11022 \times 10^{-16}$ \\
\hline 0.8 & $-9.982947758 \times 10^{-1}$ & $-9.982947758 \times 10^{-1}$ & $7.77156 \times 10^{-16}$ \\
\hline 0.9 & $-8.967584163 \times 10^{-1}$ & $-8.967584163 \times 10^{-1}$ & $2.22045 \times 10^{-16}$ \\
\hline 1.0 & $-6.536436209 \times 10^{-1}$ & $-6.536436209 \times 10^{-1}$ & $5.55112 \times 10^{-16}$ \\
\hline
\end{tabular}

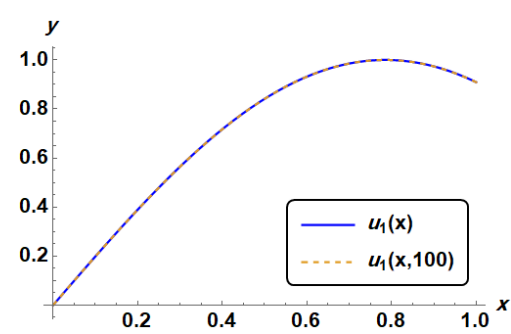

((a)) The graph of $u_{1}(x, 100)$ with the exact solution $u_{1}(x)$.

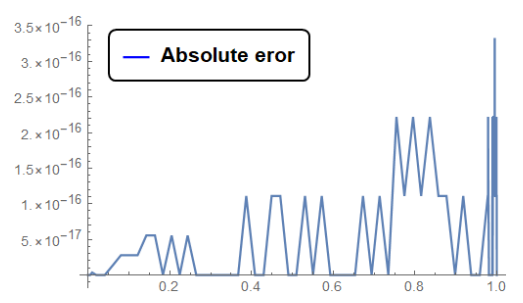

((b)) Graph of the Absolute error $\left|u_{1}(x, 100)-u_{1}(x)\right|$.

Fig. 1. Behavior of the approximate solution $u_{1}(x, 100)$ together with exact solution $u_{1}(x)$ and the Absolute error for Example 1.

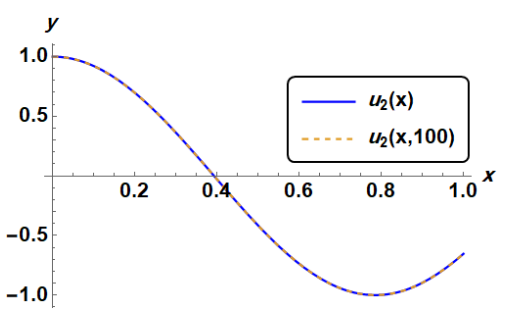

((a)) The graph of $u_{2}(x, 100)$ with the exact solution $u_{2}(x)$.

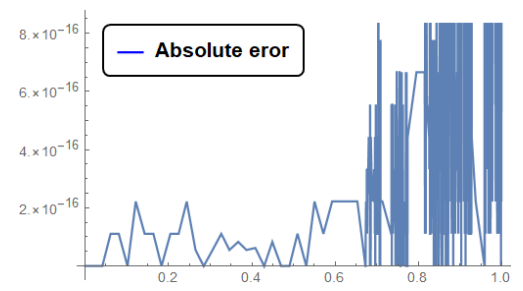

((b)) Graph of the Absolute error $\left|u_{2}(x, 100)-u_{2}(x)\right|$.

Fig. 2. Behavior of the approximate solution $u_{2}(x, 100)$ together with exact solution $u_{2}(x)$ and the Absolute error for Example 1.

The previous tables show that the approximate solution was in excellent agreement with the exact solution at some selected points. Moreover, figures 1 and 2 demonstrate that the accuracy is not limited to these points, but is consistent over the interval $[0,1]$.

Example 2: Consider the following system of linear fractional Fredholm IDEs:

$$
\begin{aligned}
& D^{\frac{1}{2}} u_{1}(x)=f_{1}(x)+\int_{0}^{1} x u_{2}(t) d t \\
& D^{\frac{1}{2}} u_{2}(x)=f_{2}(x)+\int_{0}^{1}\left(t x u_{1}(t)+x^{2} u_{3}(t)\right) d t \\
& D^{\frac{1}{2}} u_{2}(x)=f_{2}(x)+\int_{0}^{1}\left(u_{1}(t)+x u_{3}(t)\right) d t,
\end{aligned}
$$

subject to the initial condition $u_{1}(0)=1, u_{2}(0)=0$, and $u_{3}(0)=1$, where

$$
\begin{aligned}
& f_{1}(x)=\sqrt{x} E_{1, \frac{3}{2}}(x)-\frac{4 x}{3} \\
& f_{2}(x)=-x-\frac{1}{3}\left(e^{3}-1\right) x^{2}+\frac{3 \pi+32 x^{\frac{3}{2}}}{6 \sqrt{\pi}} \\
& f_{3}(x)=3 \sqrt{x} E_{1, \frac{3}{2}}(3 x)+1-e-\frac{1}{3}\left(e^{3}-1\right) x,
\end{aligned}
$$

the exact solution is $u_{1}(x)=e^{x}, u_{2}(x)=2 x^{2}+\sqrt{x}$, and $u_{3}(x)=e^{3 x}$.

We expand the solution to the system (12) as fractional power series representation about $x=0$ of the form

$$
\begin{aligned}
& u_{1}(x)=\sum_{j=0}^{\infty} c_{j} \frac{x^{\frac{j}{2}}}{\Gamma\left(\left(\frac{j}{2}\right)+1\right)} \\
& u_{2}(x)=\sum_{j=0}^{\infty} b_{j} \frac{x^{\frac{j}{2}}}{\Gamma\left(\left(\frac{j}{2}\right)+1\right)} \\
& u_{3}(x)=\sum_{j=0}^{\infty} p_{j} \frac{x^{\frac{j}{2}}}{\Gamma\left(\left(\frac{j}{2}\right)+1\right)} .
\end{aligned}
$$


Now, we apply the steps mentioned in section III by first replacing each $u_{l}(x)$ by the $k-t h$ truncated series $u_{l}(x, k)$ in system (12) for all $l=1,2,3$. Accordingly, we have

$$
\begin{aligned}
& D^{\frac{1}{2}} u_{1}(x, k)=f_{1}(x)+\int_{0}^{1} x u_{2}(t, k) d t \\
& D^{\frac{1}{2}} u_{2}(x, k)=f_{2}(x)+\int_{0}^{1}\left(t x u_{1}(t, k)+x^{2} u_{3}(t, k)\right) d t \\
& D^{\frac{1}{2}} u_{2}(x, k)=f_{2}(x)+\int_{0}^{1}\left(u_{1}(t, k)+x u_{3}(t, k)\right) d t .
\end{aligned}
$$

Here, we write down some calculations needed for step 2:

1)

$$
\begin{aligned}
& D^{\frac{1}{2}(n)} f_{1}(x)=\sum_{j=\beta_{n}}^{\infty} \frac{x^{j-\frac{n-1}{2}}}{\Gamma\left(j-\frac{n-3}{2}\right)}, \forall n \geq 3 . \text { Where } \\
& \beta_{n}= \begin{cases}\frac{n}{2} & \text { if } n=2 m, m=2,3,4 \cdots \\
\frac{n-1}{2} & \text { if } n=2 m-1, m=2,3,4 \cdots\end{cases}
\end{aligned}
$$

2) From note number one, we conclude that:

$$
D^{\frac{1}{2}(n)} f_{1}(0)=\left\{\begin{array}{l}
0 \text { if } n=2 m, m=2,3,4 \cdots \\
1 \text { if } n=2 m-1, m=2,3,4 \cdots
\end{array}\right.
$$

3)

$$
D^{\frac{1}{2}(n)} f_{2}(0)=0, \forall n \geq 5
$$

4)

$$
\begin{gathered}
D^{\frac{1}{2}(n)} f_{3}(x)=\sum_{j=\beta_{n}}^{\infty} \frac{(3)^{j+1} x^{j-\frac{n-1}{2}}}{\Gamma\left(j-\frac{n-3}{2}\right)}, \forall n \geq 3 . \text { Where } \\
\beta_{n}= \begin{cases}\frac{n}{2} & \text { if } n=2 m, m=2,3,4 \cdots \\
\frac{n-1}{2} & \text { if } n=2 m-1, m=2,3,4 \cdots\end{cases}
\end{gathered}
$$

5) From note number three, we conclude that:

$$
D^{\frac{1}{2}(n)} f_{3}(0)= \begin{cases}0 & \text { if } n=2 m, m=2,3,4 \cdots \\ 3^{\frac{n+1}{2}} & \text { if } n=2 m-1, m=2,3,4 \cdots\end{cases}
$$

6)

$$
\left.D^{\frac{1}{2}(n)}\left(\int_{0}^{1} x u_{2}(t, k) d t\right)\right|_{x=0}=0, \quad \forall n \geq 3 .
$$

7)

$$
\left.D^{\frac{1}{2}(n)}\left(\int_{0}^{1}\left(x t u_{1}(t, k)+x^{2} u_{3}(t, k)\right) d t\right)\right|_{x=0}=0, \forall n \geq 5 .
$$

8)

$$
\left.D^{\frac{1}{2}(n)}\left(\int_{0}^{1}\left(u_{1}(t, k)+x u_{3}(t, k)\right) d t\right)\right|_{x=0}=0, \quad \forall n \geq 3 .
$$

Using the initial conditions given in this example by substituting 0 in 13 , we find that $c_{0}=1, b_{0}=0$, and $p_{0}=1$. Now we write the $k \times 3$ equations as mentioned in step 2 of section III as follows:

For $m=1$, we have:

$$
\begin{gathered}
c_{1}=0, b_{1}=\frac{\sqrt{\pi}}{2} \\
p_{1}-1+e-\sum_{j=0}^{k} \frac{c_{j}}{\Gamma\left(\frac{j}{2}+2\right)}=0 .
\end{gathered}
$$

For $m=2$, we have:

$$
c_{2}=1, b_{2}=0, p_{2}=3
$$

For $m=3$, we have:

$$
\begin{array}{r}
c_{3}+\frac{4}{3}-\sum_{j=0}^{k} \frac{b_{j}}{\Gamma\left(\frac{j}{2}+2\right)}=0 \\
b_{3}+1-\sum_{j=0}^{k} \frac{(2+j) c_{j}}{2 \Gamma\left(3+\frac{j}{2}\right)}=0 \\
p_{3}+\frac{1}{3}\left(e^{3}-1\right)-\sum_{j=0}^{k} \frac{p_{m}}{\Gamma\left(2+\frac{j}{2}\right)}=0 .
\end{array}
$$

For $m=4$, we have:

$$
c_{4}=1, b_{4}=4, p_{4}=9 .
$$

For $m=5$, we have:

$$
\begin{gathered}
c_{5}=0, p_{5}=0, \\
b_{5}+\frac{2}{3}\left(e^{3}-1\right)-\sum_{j=0}^{k} \frac{2 p_{j}}{\Gamma\left(\frac{j}{2}+2\right)}=0 .
\end{gathered}
$$

Continuing in this process and using the calculations mentioned from 1 to 8 above we conclude the following:

$$
\begin{gathered}
c_{n+1}= \begin{cases}0 & \text { if } n=2 m, m=2,3,4 \cdots \\
1 & \text { if } n=2 m-1, m=2,4,6 \cdots\end{cases} \\
p_{n+1}= \begin{cases}3^{\frac{n+1}{2}} & \text { if } n=2 m-1, m=2,3,4 \cdots \\
0 & \text { if } n=2 m, m=2,3,4 \cdots\end{cases}
\end{gathered}
$$

Now, we sum up the work done above. The unknown coefficients are $p_{1}, c_{3}, b_{3}, p_{3}$, and $b_{5}$. We can easily determine the value of these unknown coefficients, by solving the above equations for them, once we chose the level of approximation (the value of $k$ ) we want. As $k$ gets larger, the approximation gets better.

If we choose $k=100$. Then we find the values of $p_{1}, c_{3}, b_{3}, p_{3}$, and $b_{5}$ by solving $(14 \mid 15[16[17|18| 19)$ for them. We conclude that $c_{3}=-7.91311 \times 10^{-45}, b_{3}=-1.70076 \times$ $10^{-45}, b_{5}=-8.60904 \times 10^{-44}, p_{1}=-2.38106 \times 10^{-45}$, and $p_{3}=-4.30452 \times 10^{-44}$. 


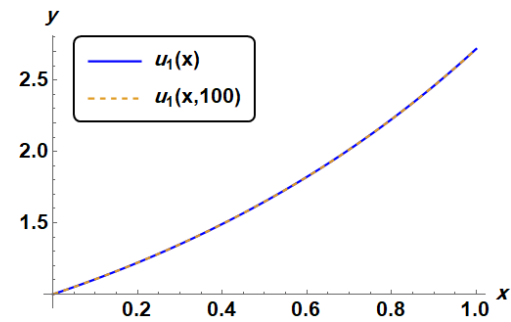

((a)) The graph of $u_{1}(x, 100)$ with the exact solution $u_{1}(x)$.

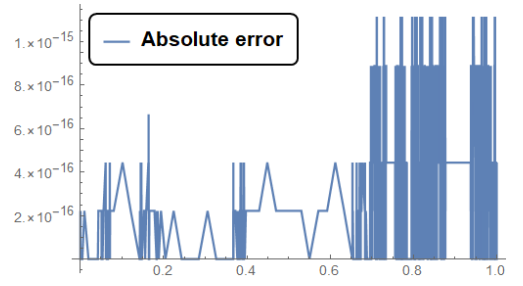

((b)) Graph of the Absolute error $\left|u_{1}(x, 100)-u_{1}(x)\right|$.

Fig. 3. Behavior of the approximate solution $u_{1}(x, 100)$ together with exact solution $u_{1}(x)$ and the Absolute error for Example 2.

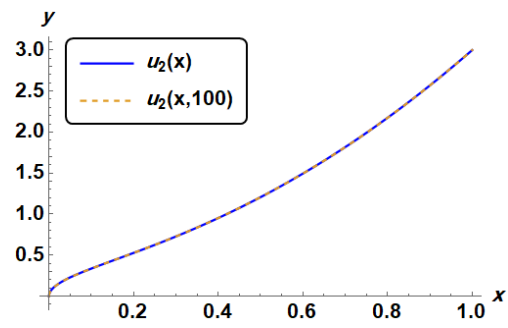

((a)) The graph of $u_{2}(x, 100)$ with the exact solution $u_{2}(x)$.

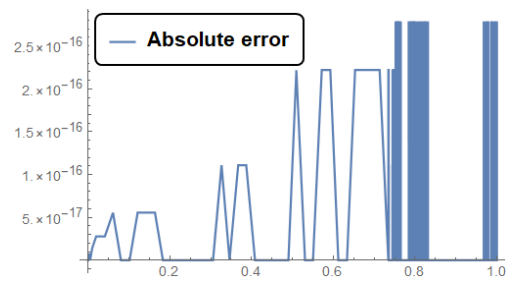

((b)) Graph of the Absolute error $\left|u_{2}(x, 100)-u_{2}(x)\right|$.

Fig. 4. Behavior of the approximate solution $u_{2}(x, 100)$ together with exact solution $u_{2}(x)$ and the Absolute error for Example 2.

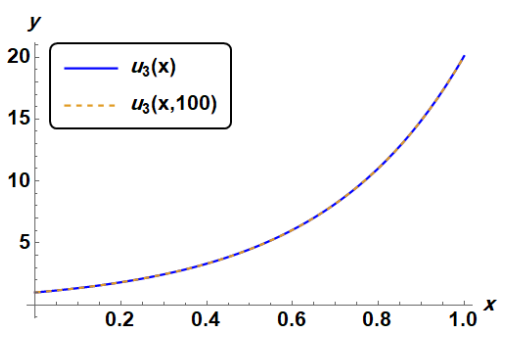

((a)) The graph of $u_{3}(x, 100)$ with the exact solution $u_{3}(x)$.

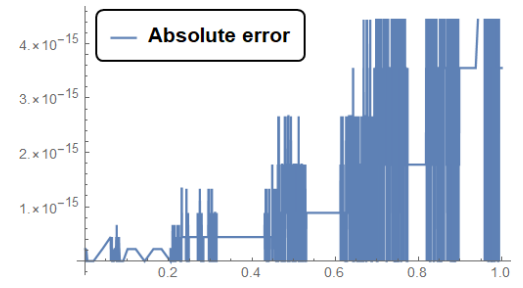

((b)) Graph of the Absolute error $\left|u_{3}(x, 100)-u_{3}(x)\right|$.

Fig. 5. Behavior of the approximate solution $u_{3}(x, 100)$ together with exact solution $u_{3}(x)$ and the Absolute error for Example 2.

\section{RESIDUAL POWER SERIES METHOD(RPSM) TO SOLVE FRACTIONAL FREDHOLM IDES}

We divide this section into two parts. In the first part, we review and correct the main ideas mentioned in paper [24] which employed the residual power series method to solve a class of fractional Fredholm IDEs, which is the single equation form of the system (1). In the second part, we compare the presented scheme in section III with the RPSM and we correct the examples in paper [24] which put the reader under misapprehension that the RPSM works effectively on fractional Fredholm IDEs which is not the case as we are going to clarify that. Finally, we solve a fractional Fredholm IDE, that has been investigated in a number of previous studies, and compare our results with the previous studies ones.

V.A Review on applying the RPSM in the paper [24]

In the paper [24], Syam applied the RPSM to solve the following class of fractional Fredholm IDEs:

$$
\begin{aligned}
D^{\alpha} u(x)=f(x) & +\lambda \int_{a}^{b} u(t) K(x, t) d t, \\
& 0<\alpha \leq 1, x \in \mathbb{R}, a \leq t \leq b
\end{aligned}
$$

subject to the initial condition $u(a)=a_{0}$. where the fractional derivative is in the Caputo sense, $a$ and $b$ are constants, $\lambda$ is a parameter, $K(x, t)$ is arbitrary continuous kernel over $[a, b]^{2}, u(x)$ is analytic unknown function to be calculated, and $f(x)$ is a smooth function. The residual power series method [1] expands the solution to (1) as fractional power series about $x=a$ of the following form:

$$
u(x)=\sum_{j=0}^{\infty} c_{j} \frac{(x-a)^{j \alpha}}{\Gamma(j \alpha+1)}
$$

Then, we approximate $u(x)$ by the $\mathrm{k}$-th truncated series $u(x, k)$ of the form:

$$
u(x, k)=\sum_{j=0}^{k} c_{j} \frac{(x-a)^{j \alpha}}{\Gamma(j \alpha+1)}
$$


using the initial condition $u(a)=a_{0}=c_{0}$, we rewrite $u(x, k)$ as:

$$
u(x, k)=a_{0}+\sum_{j=1}^{k} c_{j} \frac{(x-a)^{j \alpha}}{\Gamma(j \alpha+1)}
$$

Now, the residual functions to 20 can be defined as:

$$
\operatorname{Res}_{u}(x)=D^{\alpha} u(x)-f(x)-\lambda \int_{a}^{b} u(t) K(x, t) d t
$$

and the the k-th residual function as:

$$
\operatorname{Res}_{u, k}(x)=D^{\alpha} u(x, k)-f(x)-\lambda \int_{a}^{b} u(t, k) K(x, t) d t
$$

The fundamental properties of RPSM related to the residual functions as in [31, 35, 36] are

1) $\lim _{k \rightarrow \infty} \operatorname{Res}_{u, k}(x)=\operatorname{Res}_{u}(x)=0$, for each $x>0$.

2) $D_{x}^{(m-1) \alpha} \operatorname{Res}_{u, m}(0)=D_{x}^{(m-1) \alpha} \operatorname{Res}_{u}(0)=0$,

for $m=1,2,3, \cdots$.

To find the coefficients $c_{m}$ for $m=1,2,3, \cdots, k$, we solve the following algebraic fractional differential equations for $m=$ $1,2,3, \cdots, k$,

$$
D^{(m-1) \alpha} \operatorname{Res}_{u, m}(a)=0,
$$

where $D^{m \alpha}=D^{\alpha} \cdot D^{\alpha} \cdots D^{\alpha}(m-$ times $)$.

The author in paper [24] made some mistakes in obtaining the analytic-numeric solution $u_{k}(x)$ for 20$)$ using the RPSM. Specifically equations $(31,32)$ in his paper [24] are not correct. Thus, here we derive the right recursive formula for generating the coefficients $c_{n}$ resulted from applying the RPSM as follows.

The process of finding the coefficient $c_{m}$ requires finding the coefficients $c_{1}, c_{2}, \cdots, c_{m-1}$ recursively. Thus, for any $n=1,2,3, \cdots, k$, we first find $c_{1}, c_{2}, \cdots, c_{n-1}$ through solving algebraic fractional differential equation 23 for $m=$ $1,2,3, \cdots, n-1$. Then, we solve (23) for $m=n$ as follows:

$$
\begin{aligned}
D^{(n-1)} \operatorname{Res}_{u, n}(a)=D^{n \alpha} & u(a, n)-D^{(n-1) \alpha} f(a) \\
& -\lambda \int_{a}^{b} u(t, n) D^{(n-1) \alpha} k(a, t) d t \\
=c_{n} & -c_{n} \lambda \int_{a}^{b} \frac{(t-a)^{n \alpha} D^{(n-1) \alpha} k(a, t)}{\Gamma(1+n \alpha)} d t \\
& -D^{(n-1) \alpha} f(a) \\
& -\lambda \sum_{j=0}^{n-1} c_{j} \int_{a}^{b} \frac{(t-a)^{j \alpha} D^{(n-1) \alpha} k(a, t)}{\Gamma(1+j \alpha)} \\
& =0
\end{aligned}
$$

Therefore, for any $n=1,2,3, \cdots, k$, we have

$$
c_{n}=\frac{D^{(n-1) \alpha} f(a)+\lambda \sum_{j=0}^{n-1} c_{j} \int_{a}^{b} \frac{(t-a)^{j \alpha} D^{(n-1) \alpha} k(a, t)}{\Gamma(1+j \alpha)} d t}{1-\lambda \int_{a}^{b} \frac{(t-a)^{n \alpha} D^{(n-1) \alpha} k(a, t)}{\Gamma(1+n \alpha)} d t},
$$

accordingly, the k-th RPS approximation is

$$
\begin{aligned}
& u(x, k)=a_{0}+\sum_{n=1}^{k}\left(\left(D^{(n-1) \alpha} f(a)\right.\right. \\
&\left.+\lambda \sum_{j=0}^{n-1} c_{j} \int_{a}^{b} \frac{(t-a)^{j \alpha} D^{(n-1) \alpha} k(a, t)}{\Gamma(1+j \alpha)} d t\right) / \\
&\left.\left(1-\lambda \int_{a}^{b} \frac{(t-a)^{n \alpha} D^{(n-1) \alpha} k(a, t)}{\Gamma(1+n \alpha)} d t\right)\right) * \frac{(x-a)^{n \alpha}}{\Gamma(n \alpha+1)}
\end{aligned}
$$

V.B comparison between the RPSM and the proposed scheme

The numerical examples presented in paper [24], to show the efficiency of the RPSM in solving (20), are not correct. The right-hand side of the three fractional Fredholm IDEs $(33,38,45)$ does not equal the left-hand side of each one. In this section, we correct two of the three examples written in the paper [24] by fixing $u(x), k(x, t)$, and changing $f(x)$ for each equation that satisfies it, in addition to solving and comparing a problem addressed by many previous studies.

Example 3:[17]. Consider the following Fredholm FIDE:

$$
\begin{gathered}
D^{\frac{1}{2}} u(x)=\frac{32}{3 \sqrt{\pi}} x^{1.5}+\frac{16}{\sqrt{\pi}} x^{2.5}-2 x \\
+\int_{0}^{1} x t u(t) d t
\end{gathered}
$$

Subject to the initial condition $u(0)=0$, and exact solution $u(x)=4 x^{2}+5 x^{3}$.

We expand the solution to the equation $(25)$ as a fractional power series representation about $x=0$ in the form

$$
u(x)=\sum_{n=0}^{\infty} c_{n} \frac{x^{\frac{n}{2}}}{\Gamma\left(\frac{n}{2}+1\right)} .
$$

Using the initial conditions given in this example by substituting 0 in 26 , we find that $c_{0}=0$. Then we apply the recursive formula in (24) to find that: $\mathrm{c}_{1}=c_{2}=c_{5}=0$,

$c_{3}=\frac{-42 \sqrt{\pi}}{-8+21 \sqrt{\pi}}, c_{4}=8, c_{6}=30$,

since $D_{x}^{(n-1) \alpha} f(0)=D_{x}^{(n-1) \alpha} k(0, t)=0$,

$\forall n \geq 7$. Thus, $c_{n}=0, \forall n \geq 7$. Therefor, we find the residual power series approximation

$$
u_{R}(x, k)=\frac{56}{8-21 \sqrt{\pi}} x^{1.5}+4 x^{2}+5 x^{3}, \forall k \geq 6
$$

Now, we solve this example using the proposed scheme in section III by following the steps outlined there.

We first replace each $u(x)$ by the $k-t h$ truncated series $u(x, k)$ in 25). Accordingly, we have:

$$
D^{\frac{1}{2}} u(x, k)=f(x)+\int_{0}^{1} x t u(t, k) d t,
$$

where $f(x)=\frac{32 x^{1.5}}{3 \sqrt{\pi}}+\frac{16}{\sqrt{\pi}} x^{2.5}-2 x$. Here, we write down some 8 calculations needed for step 2: 
1)

$$
D^{\frac{1}{2}(n)} f(x)=0, \forall n \geq 6
$$

2)

$$
D^{\frac{1}{2}(n)} \int_{0}^{1} x t u(t, k) d t=0, \quad \forall n \geq 3 .
$$

Now, as stated in step 2 of section IIII we write the $k \times 1$ equations as follows:

For $m=1$, we have:

$$
c_{1}=0 .
$$

For $m=2$, we have:

$$
c_{2}=0
$$

For $m=3$, we have:

$$
c_{3}+2-\sum_{j=0}^{k} \frac{2 c_{j}}{(4+j) \Gamma\left(\frac{j}{2}+1\right)}=0
$$

Continuing in this process and using the calculations mentioned from 1 to 2 above, we conclude the following:

$$
\begin{gathered}
c_{n}=D^{\frac{1}{2(n-1)}} f(0)+\left.D^{\frac{1}{2}(n-1)}\left(\int_{0}^{1} x t u(t, k) d t\right)\right|_{x=0}=0, \\
\forall n \geq 7 .
\end{gathered}
$$

Now, we sum up the work done above. The unknown coefficient is $c_{3}$ which can be easily determined by solving the above equations for it. The value of $k$ in this example does not really matter, because $c_{n}=0, \forall n \geq 7$, as long as $k \geq 6$.

If we choose $k \geq 6$. Then we find the value of $c_{3}$ by solving $27 \mid 28$ 29|30) for them. We conclude that $c_{3}=0$.

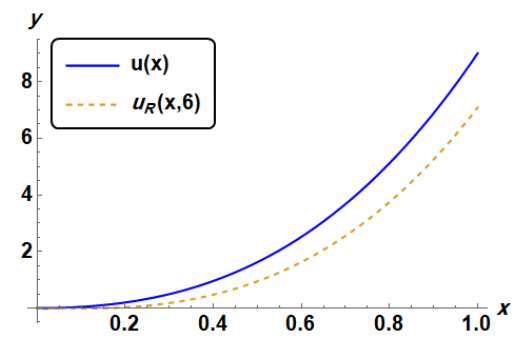

((a)) The graph of $u_{R}(x, 6)$ with the exact solution $u(x)$.

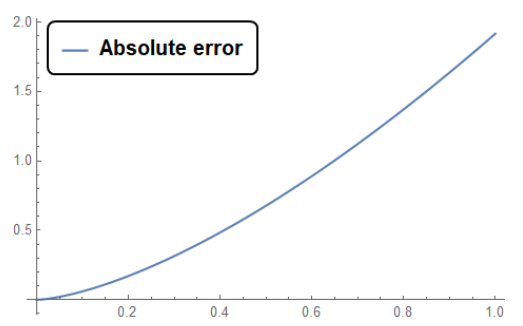

((b)) Graph of the Absolute error $\left|u_{R}(x, 6)-u(x)\right|$.

Fig. 6. Behavior of the RPSM approximate solution $u_{R}(x, 6)$ together with exact solution $u(x)$ and the Absolute error for Example 3.
Using the proposed method in section III we were able to obtain the exact solution for this example. whereas, the approximate solution in the RPSM is not suitable, according to the Absolute error graph in figure 6, which is bounded between 0 and 1.92 .

Example 4:[17]. Consider the following Fredholm FIDE:

$$
D^{\frac{1}{4}} u(x)=f(x)+\int_{0}^{1} x^{2} t u(t) d t,
$$

subject to the initial condition $u(0)=1$, where

$$
f(x)=\frac{1}{2} x^{\frac{3}{4}} E_{1, \frac{7}{4}}\left(\frac{x}{2}\right)+2(\sqrt{e}-2) x^{2},
$$

and exact solution $u(x)=e^{\frac{x}{2}}$.

We expand the solution to the equation (31) as a fractional power series representation about $x=0$ in the form

$$
u(x)=\sum_{n=0}^{\infty} c_{n} \frac{x^{\frac{n}{4}}}{\Gamma\left(\frac{n}{4}+1\right)} .
$$

Using the initial conditions given in this example by substituting 0 in (32), we find that $c_{0}=1$. Then we apply the recursive formula in (24) to find that:

$$
\begin{aligned}
& c_{1}=c_{2}=c_{3}=c_{5}=c_{6}=c_{7}=0, \\
& c_{4}=\frac{1}{2}, c_{8}=\frac{1}{4}, c_{9} \approx-1.13828 \times 10^{-2}, \\
& c_{n+1}= \begin{cases}\frac{1}{2^{\frac{n+1}{4}}} & \text { if } n=2 m-1, m=6,8,10 \cdots \\
0 & \text { if } n=2 m-1, m=5,7,9 \cdots \\
0 & \text { if } n=2 m, m=5,6,7 \ldots\end{cases}
\end{aligned}
$$

Therefore, we find the residual power series approximation $u_{R}(x, 100)=u(x, 100)$.

Now, we solve this example using the proposed scheme in section [III by applying the steps mentioned there.

We first replace each $u(x)$ by the $k-t h$ truncated series $u(x, k)$ in 31. Accordingly, we have

$$
D^{\frac{1}{4}} u(x, k)=f(x)+\int_{0}^{1} x^{2} t u(t) d t .
$$

Here, we write down some calculations needed for step 2:

1)

$$
D^{\frac{1}{4}(n)} k(0, t)=0, \forall n \geq 9
$$

2)

$$
\begin{gathered}
D^{\frac{1}{4}(n)} \frac{1}{2} x^{\frac{3}{4}} E_{1, \frac{7}{4}}\left(\frac{x}{2}\right)=\sum_{j=\beta_{n}}^{\infty} \frac{x^{j+\frac{3-n}{4}}}{2^{j+1} \Gamma\left(j+\frac{7-n}{4}\right)}, \text { where } \\
\beta_{n}= \begin{cases}\frac{n}{4} & \text { if } n=2 m, m=2,4,6 \cdots \\
\frac{n-2}{4} & \text { if } n=2 m, m=1,3,5 \cdots \\
\frac{n-3}{4} & \text { if } n=2 m-1, m=2,4,6 \cdots \\
\frac{n-1}{4} & \text { if } n=2 m-1, m=1,3,5 \cdots\end{cases}
\end{gathered}
$$


3) Using the previous note, we find that

$$
D^{\frac{1}{4}(n)} f(0)= \begin{cases}\frac{1}{2^{\frac{n+1}{4}}} & \text { if } n=2 m-1, m=6,8,10 . . \\ 0 & \text { if } n=2 m-1, m=5,7,9 . . \\ 0 & \text { if } n=2 m, m=5,6,7 \ldots\end{cases}
$$

Using the initial conditions given in this example by substituting 0 in (32), we find that $c_{0}=1$. Now we write the $k \times 1$ equations as mentioned in step 2 of section III as follows: For $m=1,2, \cdots, 8$, we find that:

$$
\begin{array}{r}
c_{1}=c_{2}=c_{3}=c_{5}=c_{6}=c_{7}=0, c_{4}=\frac{1}{2}, c_{8}=\frac{1}{4}, \\
c_{9}=4(\sqrt{e}-2)+\sum_{j=0}^{k} \frac{8 c_{j}}{(j+8) \Gamma\left(1+\frac{j}{4}\right)} .
\end{array}
$$

Continuing in this process and using the calculations mentioned from 1 to 3 above, we conclude the following for $n \geq 9$ :

$$
c_{n+1}= \begin{cases}\frac{1}{2^{\frac{n+1}{4}}} & \text { if } n=2 m-1, m=6,8,10 \cdots \\ 0 & \text { if } n=2 m-1, m=5,7,9 \cdots \\ 0 & \text { if } n=2 m, m=5,6,7 \cdots\end{cases}
$$

Now, we sum up the work done above. The unknown coefficient is $c_{9}$ which can be easily determined by solving the above equations for it once we chose the level of approximation (the value of $k$ ) we want. As $k$ gets larger, the approximation gets better. If we choose $k=100$. Then we find the value of $c_{9}$ by solving 3435 for it. We conclude that $c_{9}=-3.29558 \times 10^{-36}$.

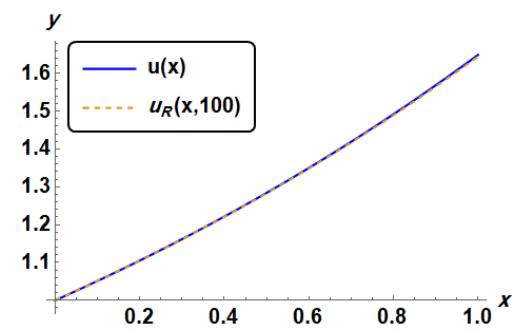

((a)) The graph of $u_{R}(x, 100)$ with the exact solution $u(x)$.

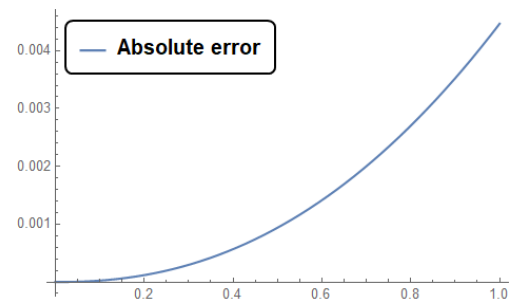

((b)) Graph of the Absolute error $\left|u_{R}(x, 100)-u(x)\right|$.

Fig. 7. Behavior of the RPSM approximate solution $u_{R}(x, 100)$ together with exact solution $u(x)$ and the Absolute error for Example 4.

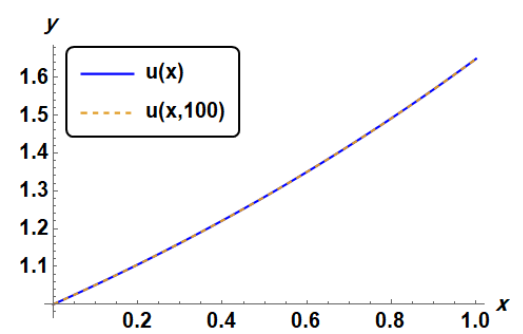

((a)) The graph of $u(x, 100)$ with the exact solution $u(x)$.

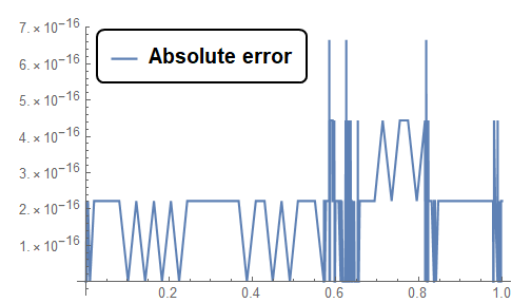

((b)) Graph of the Absolute error $|u(x, 100)-u(x)|$.

Fig. 8. Behavior of the approximate solution $u(x, 100)$ using our approach together with exact solution $u(x)$ and the Absolute error for Example 4.

The proposed method in section III yields a high-accuracy approximate solution with Absolute error bounded between 0 and $7 \times 10^{-16} \forall x \in[0,1]$ as the graph shows in figure 8 . The RPSM, on the other hand, provides a good approximation, with an Absolute error range of 0 to $4.5 \times 10^{-3}$ as shown in figure 7. However, according to the figures, the proposed scheme's approximation is much better than the RPS approximation in this example.

Example 5: Consider the following Fredholm FIDE:

$$
D^{\frac{1}{2}} u(x)=f(x)+\int_{0}^{1} x t u(t) d t,
$$

subject to the initial condition $u(0)=0$, where

$$
f(x)=\frac{\frac{8}{3} x^{\frac{3}{2}}-2 x^{\frac{1}{2}}}{\sqrt{\pi}}+\frac{x}{12},
$$

and exact solution $u(x)=x^{2}-x$.

We expand the solution to the equation (36) as a fractional power series representation about $x=0$ in the form

$$
u(x)=\sum_{n=0}^{\infty} c_{n} \frac{x^{\frac{n}{2}}}{\Gamma\left(\frac{n}{2}+1\right)} .
$$

Using the initial conditions given in this example by substituting 0 in (37), we find that $c_{0}=0$. Then we apply the recursive formula in (24) to find that:

$$
\begin{gathered}
c_{1}=0, c_{2}=-1, c_{3}=\frac{-1}{4\left(1-\frac{8}{21 \sqrt{\pi}}\right)}, c_{4}=2 \\
c_{n}=0, \forall n \geq 5 .
\end{gathered}
$$

Therefore, we find the residual power series approximation $u_{R}(x, 4)=-x-\frac{x^{\frac{3}{2}}}{3\left(\sqrt{\pi}-\frac{8}{21}\right)}+x^{2}$.

Now, we solve this example using the proposed scheme in 
section [III by applying the steps mentioned there.

We first replace each $u(x)$ by the $k-t h$ truncated series $u(x, k)$ in (36). Accordingly, we have

$$
D^{\frac{1}{2}} u(x, k)=f(x)+\int_{0}^{1} x t u(t, k) d t,
$$

Here, we write down some calculations needed for step 2:

1)

$$
D^{\frac{1}{2}(n)} f(0)=0, \forall n \geq 4
$$

2)

$$
\left.D^{\frac{1}{2}(n)}\left(\int_{0}^{1} x t u(t, k) d t\right)\right|_{x=0}=0=0, \quad \forall n \geq 3 .
$$

Now we write the $k \times 1$ equations as mentioned in step 2 of section $\amalg$ as follows:

For $m=1,2, \cdots, k$, we find that:

$$
\begin{array}{r}
c_{1}=0, c_{2}=-1, c_{4}=2, c_{3}=\frac{1}{12}+\sum_{j=0}^{k} \frac{2 c_{j}}{(j+4) \Gamma\left(1+\frac{j}{2}\right)}, \\
c_{n}=D^{\frac{1}{2}(n-1)} f(0)+\left.D^{\frac{1}{2}(n-1)}\left(\int_{0}^{1} x t u(t, k) d t\right)\right|_{x=0}=0, \forall n \geq 5 .
\end{array}
$$

Now, we sum up the work done above. The unknown coefficient is $c_{3}$ which can be easily determined by solving the above equations for it. The value of $k$ in this example does not really matter, because $c_{n}=0, \forall n \geq 5$, as long as $k \geq 4$.

If we choose $k \geq 4$. Then we find the value of $c_{3}$ by solving 39 for it. We conclude that $c_{3}=0$ and thus $u(x, 4)=$ $x^{2}-x$ which is the exact solution.

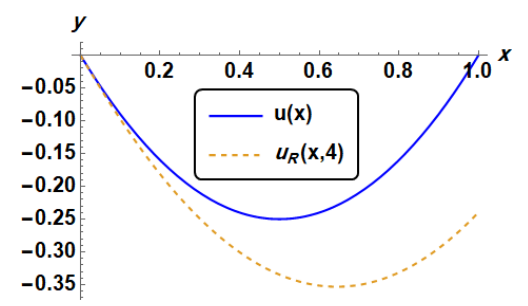

((a)) The graph of $u_{R}(x, 4)$ with the exact solution $u(x)$.

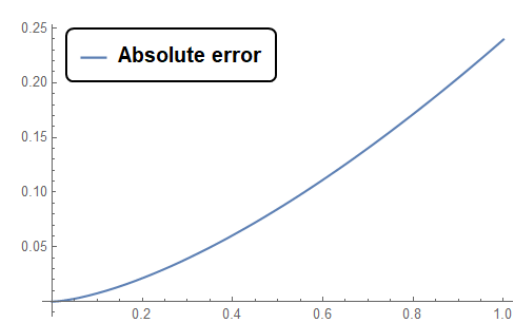

((b)) Graph of the Absolute error $\left|u_{R}(x, 4)-u(x)\right|$.

Fig. 9. Behavior of the RPSM approximate solution $u_{R}(x, 4)$ together with exact solution $u(x)$ and the Absolute error for Example 5 .

\section{Discussion AND Results}

This section presents and discusses the most prominent points that emerged from the comparison in the previous subsection V.B Furthermore, we discuss the characteristics of the proposed scheme in section III and justify why do we think that it is superior to the RPSM.

1) From looking at figure 6 part a, we can see that as $x$ gets larger the residual approximation becomes inaccurate and its graph gradually moves away from the graph of the exact solution, and unaccepted approximation at the large portion of the interval. This effect can be also seen in part $b$ of the same figure which shows that as $x$ gets larger the error increases more. which indicates a big variation between the absolute error at different points in the interval $[0,1]$ as part $b$ of that figure shows. On the other hand, we obtained the exact solution using the method in section III] Moreover, we will be able to obtain the exact solution, using the approach in section III] whenever the unknown function can be written as a finite FPS (That includes polynomials) and its highest degree is known so that we can choose the suitable level of approximation $\mathrm{k}$ that enable us to find all the coefficients.

The same results can be addressed to Example 5, which produced unaccepted approximate solution according to Figure 9 using the RPSM and exact solution using the technique in section IIII

2) From looking at figure 7 and figure 8 , we can see that both methods were able to obtain a good approximation with a better one using the approach in section [III according to the $b$ parts of each figure. Moreover, the variation in the absolute error graph of figure 8 is relatively small comparing to the RPSM ones in part $b$ of figure 7. which guarantees more stability and accuracy at each point in the interval $[0,1]$ of the approximate solution. This feature can also be seen in the figures and the tables of the first and second examples.

3) Approximate solutions had been found to the problem in example 5 using several techniques [37, 38, 39, 40, 41]. For instance, the problem was solved [37] using the Least Squares Method (LSM) and Shifted Chebyshev Polynomial by Mohammed and using the LSM and shifted Chebyshev polynomials of the third kind by Mahdy et al. [38]. Also, Mahdy et al. [39] utilized the LSM and shifted Laguerre polynomials pseudo-spectral method to solve that problem. They obtained approximate solutions to the problem in example 5 and graphed it with the exact solution as the only indicator to see how well it agreed with the exact solution, without presenting the numerical results of the errors in the problem. That is not accurate to conclude that they obtained an exact or excellent agreement with the exact solution.

Khongnual et al. in [40] found an approximate solution $u(x)=-x+x^{2}-1.57083 \times 10^{-16}(-x+$ $\left.x^{2}\right)$ using a method based on hybrid of blockpulse functions and Taylor polynomials with absolute error bounded between 0 and $4 \times 10^{-17}$. 
Also, Oyedepo et al. found an approximate solution $u_{3}(x)=0.00010800322060 .9998062943 x+$ $0.999757556 x^{2}+0.000013067 x^{3}$ and a table at some grade points that presents the absolute error bounded above by $3.5524 \times 10^{-5}$ of standard LSM and $1.4367 \times$ $10^{-4}$ of perturbed LSM Absolute error.

If we overlook some of the negatives in presenting the approximate solutions in some of the previous papers and agree on their compatibility with the exact solution, however, we have obtained the exact solution in our method. Which gives a clear indication of the importance of our method in dealing with the fractional Fredholm IDEs despite its simplicity, ease of use, and negligible computational time, unlike the numerical methods which require a major time comparing to the analytical methods.

4) We point out that the comparison between the technique in section III and the RPSM was done at the same level of approximation $(k)$.

5) The limitation of using the approach in section III is clarified in the restrictions written on system 1 in section [ in addition to that each $f_{i}(x)$ can be written as a FPS of powers $n \alpha_{i}$.

6) The proposed technique may be extended and tested to solve other classes of linear or nonlinear IDEs.

\section{CONCLUSION}

In this article, a new technique has been utilized to find an analytic-numeric solution for a system of fractional Fredholhm IDEs. Two examples were solved using the technique illustrated in section IIII where the obtained solutions were in excellent agreement with the exact solution, and better results can be obtained as $k$ gets bigger. Moreover, the main ideas in the paper [24] were reviewed. In particular, correcting the derivation of the recursive formula, which generates the unknown coefficients $c_{n}$ of the FPS, was done. Furthermore, the first two examples in the paper [24] were corrected and solved using the RPSM and the proposed technique in section III. The Absolute error figures for these two examples show high accuracy and stability in the obtained solution using the proposed method. The comparison in the figures shows a clear superiority of our method over the RPSM for solving this class of equations as explained in detail in the previous section. Also, a fractional Fredholm IDE, which was investigated in a number of previous studies, has been solved in subsection $\mathrm{VI}$ and compared the obtained solution in our approach with the previous studies ones where the obtained solution in our approach was the exact one and was the best solution between all the mentioned studies.

The reason for comparing our method with RPSM is due to the similarity of the two schemes. That is, the two schemes follow the same steps outlined in section III except that the RPSM replace each $m$ by $k$ in step 2 and replace the system 5 by the system of residual functions. But still applying the $\left.D^{(k-1) \alpha_{i}}\right|_{x=0}$ for all $i=1,2, \cdots, n$ on both sides of the equations of the system (5) result the same as applying it on the system of the residual functions $\forall k=1,2, \cdots, k$.

\section{REFERENCES}

[1] Yang, X.J., Machado, J.T., Cattani, C. and Gao, F., 2017. On a fractal LC-electric circuit modeled by local fractional calculus. Communications in Nonlinear Science and Numerical Simulation, 47, pp.200-206.

[2] El-Misiery, A.E.M. and Ahmed, E., 2006. On a fractional model for earthquakes. Applied Mathematics and Computation, 178(2), pp.207-211.

[3] He, J.H., 1999. Some applications of nonlinear fractional differential equations and their approximations. Bull. Sci. Technol, 15(2), pp.86-90.

[4] Bagley, R.L. and Torvik, P.J., 1983. A theoretical basis for the application of fractional calculus to viscoelasticity. Journal of Rheology, 27(3), pp.201-210.

[5] Bohannan, G.W., 2008. Analog fractional order controller in temperature and motor control applications. Journal of Vibration and Control, 14(9-10), pp.14871498.

[6] Rossikhin, Y.A. and Shitikova, M.V., 1997. Applications of fractional calculus to dynamic problems of linear and nonlinear hereditary mechanics of solids. Appl. Mech. Rev., 50(1), pp.15-67.

[7] Butkovskii, A.G., Postnov, S.S. and Postnova, E.A., 2013. Fractional integro-differential calculus and its control-theoretical applications. II. Fractional dynamic systems: modeling and hardware implementation. Automation and Remote Control, 74(5), pp.725-749.

[8] Pinto, D.J. and Ermentrout, G.B., 2001. Spatially structured activity in synaptically coupled neuronal networks: II. Lateral inhibition and standing pulses. SIAM Journal on Applied Mathematics, 62(1), pp.226-243.

[9] Dehghan, M., 2006. Solution of a partial integrodifferential equation arising from viscoelasticity. International Journal of Computer Mathematics, 83(1), pp.123129.

[10] Saeedi, H., Moghadam, M.M., Mollahasani, N. and Chuev, G.N., 2011. A CAS wavelet method for solving nonlinear Fredholm integro-differential equations of fractional order. Communications in Nonlinear Science and Numerical Simulation, 16(3), pp.1154-1163.

[11] Saeedi, H. and Samimi, F., 2012. He's homotopy perturbation method for nonlinear ferdholm integrodifferential equations of fractional order. International Journal of Engineering Research and Applications, 2(5), pp.52-56.

[12] Saeedi, H., 2013. Application of Haar wavelets in solving nonlinear fractional Fredholm integro-differential equations. J. Mahani Math. Res. Center, 2(1), pp.15-28.

[13] Setia, A., Liu, Y. and Vatsala, A.S., 2014. Solution of linear fractional Fredholm integro-differential equation by using second kind Chebyshev wavelet. International Conference on Information Technology: New Generations,pp. 465-469. 
[14] Darweesh, A., Alquran, M., and Aghzawi, K., 2020. New Numerical Treatment for a Family of TwoDimensional Fractional Fredholm Integro-Differential Equations. Algorithms, 18, pp.1-18.

[15] Adams, E. and Spreuer, H., 1975. Uniqueness and stability for boundary value problems with weakly coupled systems of nonlinear integro-differential equations and application to chemical reactions. Journal of Mathematical Analysis and Applications, 49(2), pp.393-410.

[16] Holmåker, K., 1993. Global asymptotic stability for a stationary solution of a system of integro-differential equations describing the formation of liver zones. SIAM Journal on Mathematical Analysis, 24(1), pp.116-128.

[17] Momani, S. and Qaralleh, R., 2006. An efficient method for solving systems of fractional integro-differential equations. Computers and Mathematics with Applications, 52(3-4), pp.459-470.

[18] Khader, M.M. and Sweilam, N.H., 2013. On the approximate solutions for system of fractional integrodifferential equations using Chebyshev pseudo-spectral method. Applied Mathematical Modelling, 37(24), pp.9819-9828.

[19] Khalil, H. and Khan, R.A., 2015. Numerical scheme for solution of coupled system of initial value fractional order Fredholm integro differential equations with smooth solutions. Journal of Mathematical Extension, 9(2), pp.39-58.

[20] Wang, J., Xu, T.Z., Wei, Y.Q. and Xie, J.Q., 2018. Numerical simulation for coupled systems of nonlinear fractional order integro-differential equations via wavelets method. Applied Mathematics and Computation, 324(2018), pp.36-50.

[21] Mahdy, A.M.S., 2018. Numerical studies for solving fractional integro-differential equations. Journal of Ocean Engineering and Science, 3(2), pp.127-132.

[22] Mohammed, O.H. and Malik, A.M., 2019. A modified computational algorithm for solving systems of linear integro-differential equations of fractional order. Journal of King Saud University-Science, 31(4), pp.946-955.

[23] Xie, J., Wang, T., Ren, Z., Zhang, J. and Quan, L., 2019. Haar wavelet method for approximating the solution of a coupled system of fractional-order integral-differential equations. Mathematics and Computers in Simulation, 163(2019), pp.80-89.

[24] Syam, M.I., 2017. Analytical solution of the fractional Fredholm integrodifferential equation using the fractional residual power series method. Complexity, 2017(1), pp.1-6

[25] Arqub, O.A., 2013. Series solution of fuzzy differential equations under strongly generalized differentiability. Journal of Advanced Research in Applied Mathematics, 5(1), pp. 31-52.
[26] Al-Smadi, M., 2013. Solving initial value problems by residual power series method. Theoretical Mathematics and Applications, 3(1), pp.199-210.

[27] Yao, J.J., Kumar, A. and Kumar, S., 2015. A fractional model to describe the Brownian motion of particles and its analytical solution. Advances in Mechanical Engineering, 7(12), pp.1-11.

[28] Kumar, S., Kumar, A. and Baleanu, D., 2016. Two analytical methods for time-fractional nonlinear coupled Boussinesq-Burger's equations arise in propagation of shallow water waves. Nonlinear Dynamics, 85(2), pp.699-715.

[29] Kumar, A., Kumar, S. and Yan, S.P., 2017. Residual power series method for fractional diffusion equations. Fundamenta Informaticae, 151(1-4), pp.213-230.

[30] Komashynska, I., Al-Smadi, M., Ateiwi, A. and AlObaidy, S., 2016. Approximate analytical solution by residual power series method for system of Fredholm integral equations. Applied Mathematics and Information Sciences, 10(3), pp.1-11.

[31] Alshammari, S., Al-Smadi, M., Hashim, I. and Alias, M.A., 2019. Applications of fractional power series approach in solving fractional Volterra integro-differential equations. AIP Conference Proceedings, 2111(1), pp.17.

[32] Alshammari, S., Al-Smadi, M., Hashim, I. and Alias, M.A., 2019. Analytical Approach to Mixed IntegroDifferential Equations of Fractional Order Using Power Series Expansion Principle. ASM Science Journal, 12(6), pp.47-52.

[33] Ishteva, M.A.R.I.Y.A., Boyadjiev, L.Y.U.B.O.M.I.R. and Scherer, R.U.D.O.L.F., 2005. On the Caputo operator of fractional calculus and C-Laguerre functions. Mathematical Sciences Research Journal, 9(6), pp. 1-14.

[34] El-Ajou, A., Arqub, O., Zhour, Z. and Momani, S., 2013. New results on fractional power series: theories and applications. Entropy, 15(12), pp. 5305-5323.

[35] Saadeh, R., Al-Smadi, M., Gumah, G., Khalil, H. and Khan, R.A., 2016. Numerical investigation for solving two-point fuzzy boundary value problems by reproducing kernel approach. Applied Mathematics and Information Sciences, 10(6), pp.1-13.

[36] Arqub, O.A. and Al-Smadi, M., 2014.Numerical algorithm for solving two-point, second-order periodic boundary value problems for mixed integro-differential equations. Applied Mathematics and Computation, 243, pp.911-922.

[37] D. Sh. Mohammed, 2014. Numerical solution of fractional integro-differential equations by least squares method and shifted Chebyshev polynomial. Mathematical problems in engineering, 2014. 
[38] A.M.S. Mahdy, E.M.H. Mohamed, and G.M.A. Marai, 2016. Numerical solution of fractional integrodifferential equations by least squares method and shifted Chebyshev polynomials of the third kind method. Theoretical Mathematics Applications, 6(4), pp.87-101.

[39] Rana T. Shwayyea , Amr M.S. Mahdy, 2016. Numerical solution of fractional integro-differential equations by least squares method and shifted Laguerre polynomials pseudo-spectral method. IJSER, 4, pp.1589-1596.

[40] Nattinee Khongnual, and Weerachai Thadee, 2021. Solutions of Fredholm integro-differential equations by using a hybrid of block-pulse functions and Taylor polynomials. Songklanakarin Journal of Science Technology, 43(1).

[41] Oyedepo T, Taiwo OA, Abubakar JU, and Ogunwobi ZO, 2016. Numerical studies for solving fractional integro-differential equations by using least squares method and bernstein polynomials. Fluid Mechanics Open Access, 3(3), pp.1-7.

Mudaffer Ali Alnobani was born in Jerash, Jordan, in October 3, 1996. He has got his Master's and Bachelor's degrees, both in Mathematics, from Jordan University of Science and Technology in 2018 and 2020, respectively. He worked as a teacher assistant at Jordan university of science and technology for two years. He also published his Master's thesis, "Fractional Power Series Method and Systems of Fractional Volterra Integro Differential Equations” at JUST University Library database.

Omar Abu Al Yaqin was born in Irbid, Jordan. He has a Master's and Bachelor's degrees in mathematics from Jordan University of Science and Technology in 2018 and 2020 , respectively. He was the first on the Bachelor's regiment that graduated in 2018. He has worked as mathematics teaching assistant at Jordan University of Science and Technology for two years. His research interests are in Applied mathematics and he has published his master's thesis, "Haar Wavelet Method For Solving Coupled Systems Of Fractional Fredholm Integro-Differential Equations and Factional Mixed Volterra-Fredholm Integro-Differential Equations" at Jordan University of Science and Technology library database.

\section{Creative Commons Attribution License 4.0 (Attribution} 4.0 International, CC BY 4.0)

This article is published under the terms of the Creative Commons Attribution License 4.0

https://creativecommons.org/licenses/by/4.0/deed.en_US 